PROCEEDINGS OF THE

AMERICAN MATHEMATICAL SOCIETY

Volume 132, Number 7, Pages 2159-2162

S 0002-9939(04)07241-7

Article electronically published on February 12, 2004

\title{
ON CONTRACTIBLE POLYHEDRA THAT ARE NOT SIMPLY CONTRACTIBLE
}

\author{
UMED H. KARIMOV AND DUŠAN REPOVŠ \\ (Communicated by Alan Dow)
}

\begin{abstract}
In answer to a question of Michael, Dydak, Segal and Spież have constructed a contractible polyhedron that is not strictly contractible. In the present note we prove a related result; by using alternative methods we show that there exist contractible polyhedra that are not simply (hence not strictly) contractible.
\end{abstract}

\section{INTRODUCTION}

Michael [6] introduced and investigated the concept of strict contractibility. The space $X$ is said to be strictly contractible to the point $x_{0} \in X$ if there exists a homotopy $H: X \times I \rightarrow X$ (here $I$ denotes the segment $[0,1]$ ) such that:

(a) For every $x \in X, H(x, 0)=x$ and $H(x, 1)=x_{0}$.

(b) If $H(x, t)=x_{0}$, then $x=x_{0}$ or $t=1$.

(c) For all $t, H\left(x_{0}, t\right)=x_{0}$.

If only conditions (a) and (b) hold, then a space $X$ is said to be simply contractible to the point $x_{0} \in X$.

Clearly, every strictly contractible space is also simply contractible. However, the converse does not hold: Consider the following compactum, usually called the Comb Space (see, e.g., Example 1.4.8 in [8]):

$$
E=\left(\left\{1, \frac{1}{2}, \frac{1}{3}, \ldots, 0\right\} \times I\right) \cup(I \times\{0\}) .
$$

Then $E$ is not strictly contractible to the point $x_{0}=(0,1) \in E$, but it is simply contractible to $x_{0}$.

Michael formulated the following interesting question:

Problem 1.1. Does there exist a contractible $A R$ space $X$ that is not strictly contractible to one of its points $x_{0} \in X$ ?

Recently, Dydak, Segal and Spież [2] have answered Problem 1.1 in the affirmative. The purpose of the present note is to prove, by applying the methods developed in [5], that compact contractible polyhedra considered in [2], 5] are not simply (hence not strictly) contractible.

Received by the editors April 30, 2002 and, in revised form, January 28, 2003 and April 4, 2003.

2000 Mathematics Subject Classification. Primary 55M15; Secondary 54G20.

Key words and phrases. Absolute retract, polyhedron, strong retract, strict contractibility, simple contractibility, collapsibility. 
Theorem 1.2. There exists a compact polyhedron $X$ and a point $v_{0} \in X$ such that

(i) $X$ is contractible;

(ii) $X$ is not simply contractible to $v_{0}$; and

(iii) $X$ is not strictly contractible to $v_{0}$.

\section{Preliminaries}

The suspension $\Sigma Z$ of a space $Z$ is the quotient space of the product $Z \times I$ in which the subspaces $Z \times\{0\}$ and $Z \times\{1\}$ are identified to points $v_{0}$ and $v_{1}$, respectively, and are called the vertices of $\Sigma Z$.

Let $p: Z \times I \rightarrow \Sigma Z$ be the quotient mapping, $p_{1}: Z \times I \rightarrow Z$ and $p_{2}: \Sigma Z \rightarrow I$ be the canonical projections. Obviously, the natural mapping $p^{-1}: \Sigma Z \rightarrow Z \times I$ is a multivalued mapping.

Definition 2.1. A mapping $g: \Sigma Z \rightarrow \Sigma Z$ is said to be flat if $\left(p_{2} \circ g \circ p\right)\left(z_{1}, \tau\right)=$ $\left(p_{2} \circ g \circ p\right)\left(z_{2}, \tau\right)$ for every pair of points $z_{1}, z_{2} \in Z$ and every $\tau \in I$.

Definition 2.2. A homotopy $H: \Sigma Z \times I \rightarrow \Sigma Z$ is said to be flat if for every $t \in I$, the mapping $H\left({ }_{-}, t\right): \Sigma Z \rightarrow \Sigma Z$ is a flat mapping (cf. [5]).

Lemma 2.3. Let $Z$ be a compact space, $f: \Sigma Z \rightarrow \Sigma Z$ a homotopically trivial flat mapping and $H: \Sigma Z \times I \rightarrow \Sigma Z$ a homotopy between $f$ and a constant mapping. Suppose that for no fixed $\tau, t \in I$, the set $\{H(p(z, \tau), t) \mid z \in Z\}$ contains both vertices $v_{0}$ and $v_{1}$. Then there exists a flat homotopy $H^{\prime}: \Sigma Z \times I \rightarrow \Sigma Z$ from $f$ to the constant mapping.

Proof. Let $a(\tau, t)$ and $b(\tau, t)$ be the minimum and the maximum of the function $p_{2}(H(p(-, \tau), t)): Z \rightarrow I$ for given numbers $\tau$ and $t$, respectively. Define the mapping $H^{\prime}: \Sigma Z \times I \rightarrow \Sigma Z$ by the following formula:

$$
H^{\prime}(p(z, \tau), t)=p\left(p_{1} p^{-1}(H(p(z, \tau), t)), \frac{a(\tau, t)}{1+a(\tau, t)-b(\tau, t)}\right) .
$$

The set $p^{-1}(H(p(z, \tau), t))$ is not a singleton only in the case when $H(p(z, \tau), t)=v_{0}$ or $H(p(z, \tau), t)=v_{1}$. In these cases we have $a(\tau, t)=0$ and $b(\tau, t)=1$, respectively. Thus the mapping $H^{\prime}$ is well defined and obviously has the required properties (cf. [5]).

\section{Proofs}

Proposition 3.1. Let $Z$ be any noncontractible compact metric space such that $\Sigma Z$ is contractible. Let $H: \Sigma Z \times I \rightarrow \Sigma Z$ be any contraction to a point. Then there exist points $z_{0}, z_{1} \in Z$ and numbers $\tau_{0}, t_{0} \in I$ such that $H\left(p\left(z_{0}, \tau_{0}\right), t_{0}\right)=v_{0}$ and $H\left(p\left(z_{1}, \tau_{0}\right), t_{0}\right)=v_{1}$.

Proof. Suppose that there did not exist points $z_{0}, z_{1}$ in $Z$ and numbers $\tau, t$ such that

$$
H\left(p\left(z_{0}, \tau\right), t\right)=v_{0} \quad \text { and } \quad H\left(p\left(z_{1}, \tau\right), t\right)=v_{1} .
$$

By Lemma 2.3 there would then exist a flat homotopy $H: \Sigma Z \times I \rightarrow \Sigma Z$ that would connect the identity mapping to the constant one.

There corresponds to $H$ a mapping $h: I^{2} \rightarrow I$ such that

$$
h(\tau, t)=p_{2} H(p(z, \tau), t), z \in Z .
$$


Note that $p_{2} H(p(z, \tau), t)$ does not depend on $z$ since $H$ is a flat mapping.

Let $P_{i}: I^{2} \rightarrow I, i \in\{1,2\}$ be the projections $P_{1}(\tau, t)=\tau$ and $P_{2}(\tau, t)=t$. Let $l:[0,1] \rightarrow I^{2}$ be a path with $l(0)=\left(\tau_{0}, 0\right)$, where $\tau_{0} \in(0,1)$, with $l(1) \in$ $\partial\left(I^{2}\right) \backslash[0,1] \times\{0\} \quad$ (here $\partial\left(I^{2}\right)$ denotes the boundary of the square $\left.I^{2}\right)$ and which does not intersect with $h^{-1}(\{0\}) \cup h^{-1}(\{1\})$. Such paths exist since $h^{-1}(\{0\})$ and $h^{-1}(\{1\})$ are closed disjoint sets (cf. [5]).

Consider the cone $C\left(Z, \tau_{0}\right)=\left\{p(z, \tau) \mid z \in Z, \tau \geq \tau_{0}\right\} \subset \Sigma Z$ and define a mapping $g: C\left(Z, \tau_{0}\right) \rightarrow \Sigma Z \backslash\left\{v_{0}, v_{1}\right\}$ as follows:

$$
g(p(z, \tau))=H\left(p\left(z, P_{1} l\left(\frac{\tau-\tau_{0}}{1-\tau_{0}}\right)\right), P_{2} l\left(\frac{\tau-\tau_{0}}{1-\tau_{0}}\right)\right) .
$$

Identify the base $p\left(Z, \tau_{0}\right)$ of the cone $C\left(Z, \tau_{0}\right)$ with $Z$. Then the restriction $\left.g\right|_{Z}$ is an inessential mapping of $Z$ to $\Sigma Z \backslash\left\{v_{0}, v_{1}\right\}$ since every cone is contractible. However, its composition with the natural projection $\Sigma Z \backslash\left\{v_{0}, v_{1}\right\} \rightarrow Z$ is the identity mapping on $Z$. This contradicts the noncontractibility of the space $Z$.

Proof of Theorem 1.2. Let $P$ be any acyclic noncontractible polyhedron. Take, for example, the 2-dimensional polyhedron constructed in the standard way (see, e.g., 4]) from one of the following presentations (cf. [1]):

$$
\left\{a, b \mid b^{-2} a b a, b^{-3 k} a^{6 k-1}\right\}, \quad k= \pm 1,2,3 \ldots,
$$

or (cf. [3]):

$$
\left\{a_{1}, \ldots, a_{r} \mid a_{1} a_{2} a_{1}^{-1} a_{2}^{-2}, a_{2} a_{3} a_{2}^{-1} a_{3}^{-2}, \ldots, a_{r} a_{1} a_{r}^{-1} a_{1}^{-2}\right\}, \quad r>3 .
$$

Then by the Mayer-Vietoris exact sequence and by the Seifert-van Kampen theorem, the suspension $\Sigma P$ is an acyclic simply connected polyhedron. It follows by the Hurewicz theorem that all homotopy groups $\pi_{*}(\Sigma P)$ are trivial and hence $\Sigma P$ is a contractible space.

Let $v_{0}$ be a vertex of the suspension $\Sigma P$, and let $H: \Sigma P \times I \rightarrow \Sigma P$ be any homotopy between the identity mapping and the constant mapping to the point $v_{0}$. Since $P$ is a noncontractible compact polyhedron, there exist by Proposition 3.1 points $z_{0}, z_{1} \in \Sigma P$ and numbers $\tau_{0}, t_{0} \in I$ such that $H\left(p\left(z_{0}, \tau_{0}\right), t_{0}\right)=v_{0}$ and $H\left(p\left(z_{1}, \tau_{0}\right), t_{0}\right)=v_{1}$. Since $v_{0} \neq v_{1}$ it follows that $t_{0} \neq 1$. If $\tau_{0}=0$ or 1 , then $p\left(z_{0}, \tau_{0}\right)=p\left(z_{1}, \tau_{0}\right)$ and $v_{0}=v_{1}$. Hence $\tau_{0} \in(0,1)$ and $p\left(z_{0}, \tau_{0}\right) \neq v_{0}$. However, $H\left(p\left(z_{0}, \tau_{0}\right), t_{0}\right)=v_{0}$. Therefore, $X=\Sigma P$ is not simply contractible to the point $v_{0}$.

\section{ACKNOWLEDGMENTS}

We acknowledge the support by the Ministry of Education, Science and Sport of the Republic of Slovenia research program No. 0101-509 and research grants No. SLO-KIT 04-14-2002 and SLO-US-2002-01. We thank the referee for comments and suggestions.

\section{REFERENCES}

[1] W. H. Beckman, A certain class of nonaspherical 2-complexes, J. Pure Appl. Algebra 16 (1980), 243-244. MR 81d:57003

[2] J. Dydak, J. Segal, and S. Spież, On questions of strict contractibility, Topology Appl. 120 (2002), 67-75. MR 2003c:55010

[3] G. Higman, A finitely generated infinite simple group, J. London Math. Soc. 26 (1951), 61-64. MR 12:390c 
[4] C. Hog-Angeloni and W. Metzler, Geometric aspects of two-dimensional complexes, Twodimensional Homotopy and Combinatorial Group Theory, London Math. Soc. Lecture Notes 197, Cambridge University Press, Cambridge, 1993, pp. 1-50.

[5] U. H. Karimov and D. Repovš, On suspensions of noncontractible compacta of trivial shape, Proc. Amer. Math. Soc. 127 (1999), 627-632. MR 99c:54023

[6] E. Michael, Closed retracts and perfect retracts, Topology Appl. 121 (2002), 451-468. MR 2003i:54015

[7] H. Seifert and W. Threlfall, A Textbook of Topology, Pure and Appl. Math., vol. 89, Academic Press, New York, 1980. MR 82b:55001

[8] E. H. Spanier, Algebraic Topology, Springer-Verlag, New York, 1966; Corrected reprint, 1981. MR 83i:55001

Institute of Mathematics, Academy of Sciences Tajikistan, Ul. Ainy $299^{A}$, Dushanbe, TAJIKISTAN 734063

E-mail address: umed-karimov@mail.ru

Institute of Mathematics, Physics and Mechanics, University of Ljubljana, P.O. Box 2964, Luubluana, Slovenia 1001

E-mail address: dusan.repovs@uni-lj.si 